\title{
CTDAS-Lagrange v1.0: A high-resolution data assimilation system for regional carbon dioxide observations
}

Wei He ${ }^{1,2}$, Ivar R. van der Velde ${ }^{3,4}$, Arlyn E. Andrews ${ }^{3}$, Colm Sweeney ${ }^{3,4}$, John Miller ${ }^{3}$, Pieter Tans ${ }^{3}$, Ingrid T. van der Laan-Luijkx ${ }^{5,6}$, Thomas Nehrkorn ${ }^{7}$, Marikate Mountain ${ }^{7}$, Weimin Ju ${ }^{1}$, Wouter 5 Peters $^{2,5}$, Huilin Chen ${ }^{2,4}$

${ }^{1}$ International Institute for Earth System Science, Nanjing University, Nanjing, China

${ }^{2}$ Center for Isotope Research (CIO), Energy and Sustainability Research Institute Groningen (ESRIG), University of Groningen, Groningen, 9747 AG, The Netherlands

${ }^{3}$ Global Monitoring Division, NOAA Earth System Research Laboratory, Boulder, Colorado, USA

$10{ }^{4}$ Cooperative Institute for Research in Environmental Sciences (CIRES), University of Colorado, Boulder, Colorado, USA

${ }^{5}$ Department of Meteorology and Air Quality, Wageningen University, Wageningen, The Netherlands

${ }^{6}$ Utrecht University, Institute for Marine and Atmospheric Research, Utrecht University, the Netherlands

${ }^{7}$ Atmospheric and Environmental Research, Lexington, MA, USA

Correspondence to: Huilin Chen (Huilin.Chen@rug.nl)

15 To investigate the system performance at the ecoregion scale, we analyzed the prior and optimized fluxes for each aggregated Olson ecoregion types when performing the runs using different prior BCs (including the different settings "fluxonly" and "flux+BC") and different prior net biosphere fluxes (including fluxes simulated by different biosphere models and a series of modified SiBCASA fluxes) shown in Figs. S1-4. 


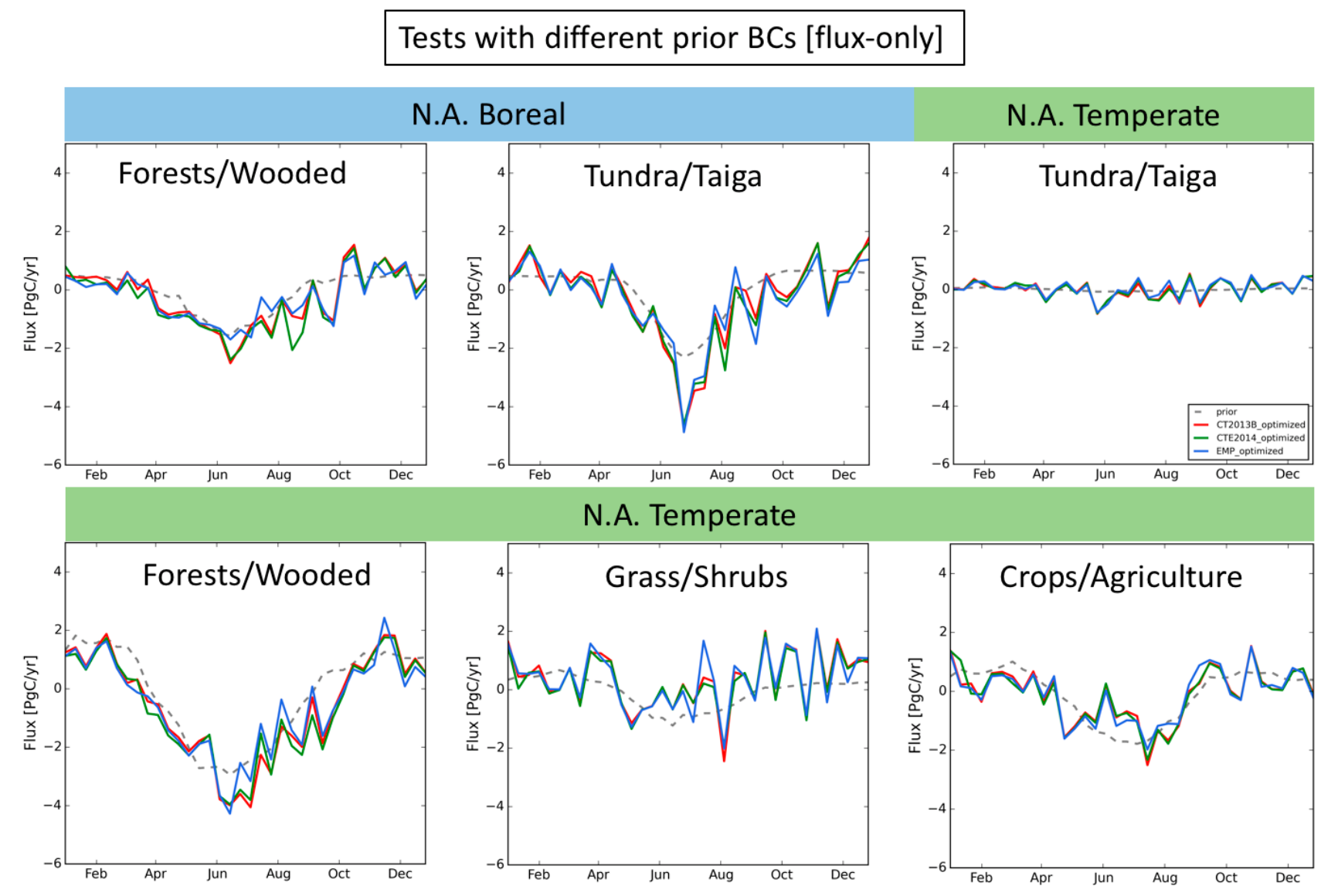

Figure S1. The time series of prior and optimized net biosphere fluxes of major aggregated ecoregions for the "flux-only" runs using three different prior BCs (CT2013B, CTE2014, and EMP). The differences caused by different prior BCs are significant in optimized fluxes per aggregated Olson ecosystem types, e.g. the deviations in the Boreal Forests/Wooded during June-September, the Temperate

5 Forests/Wooded during June-July and the Temperate Grass/Shrubs during June-July. 


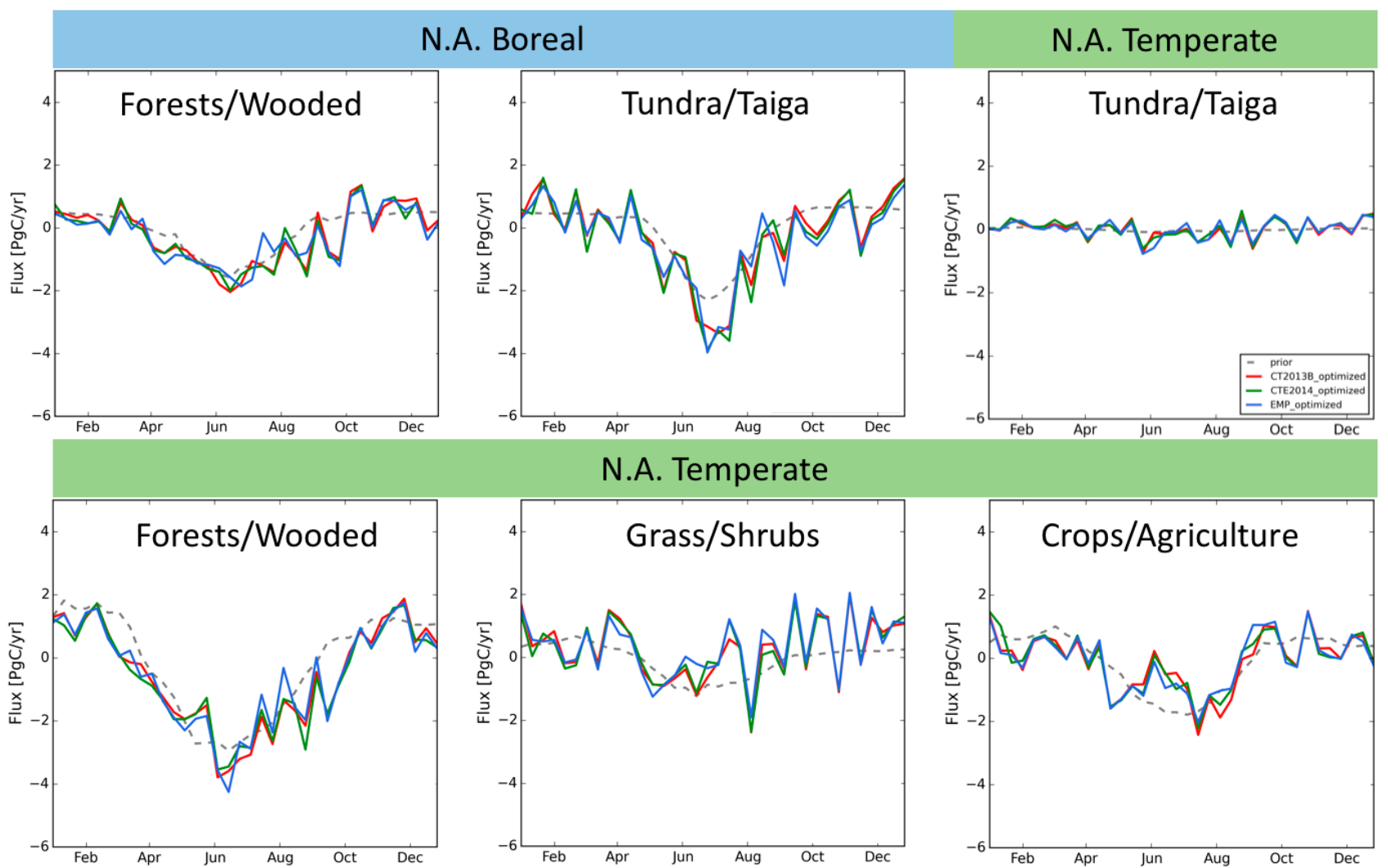

Figure S2. The time series of prior and optimized net biosphere fluxes of major aggregated ecoregions for the runs "flux $+\mathrm{BC} " \mathrm{using}$ three different prior BCs (CT2013B, CTE2014 and EMP). Compared with the results shown in Figure S1, the additional BC optimization further constrains the magnitude of the fluxes, e.g. the August - September uptake by Boreal Forests/Wooded is reduced for the runs with the prior BC CTE2014. 


\section{Tests with different prior fluxes}

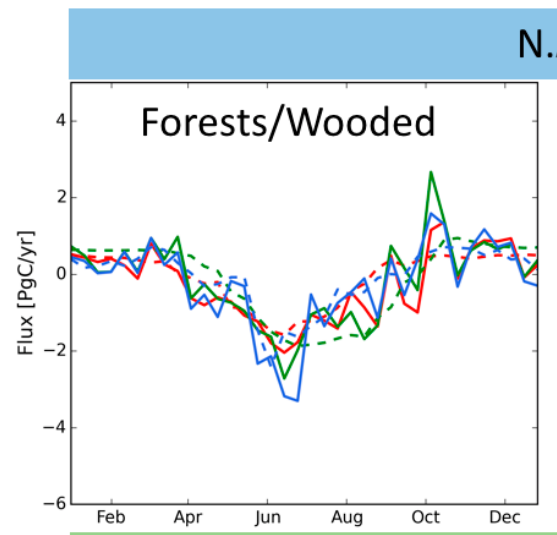

N.A. Boreal

N.A. Temperate
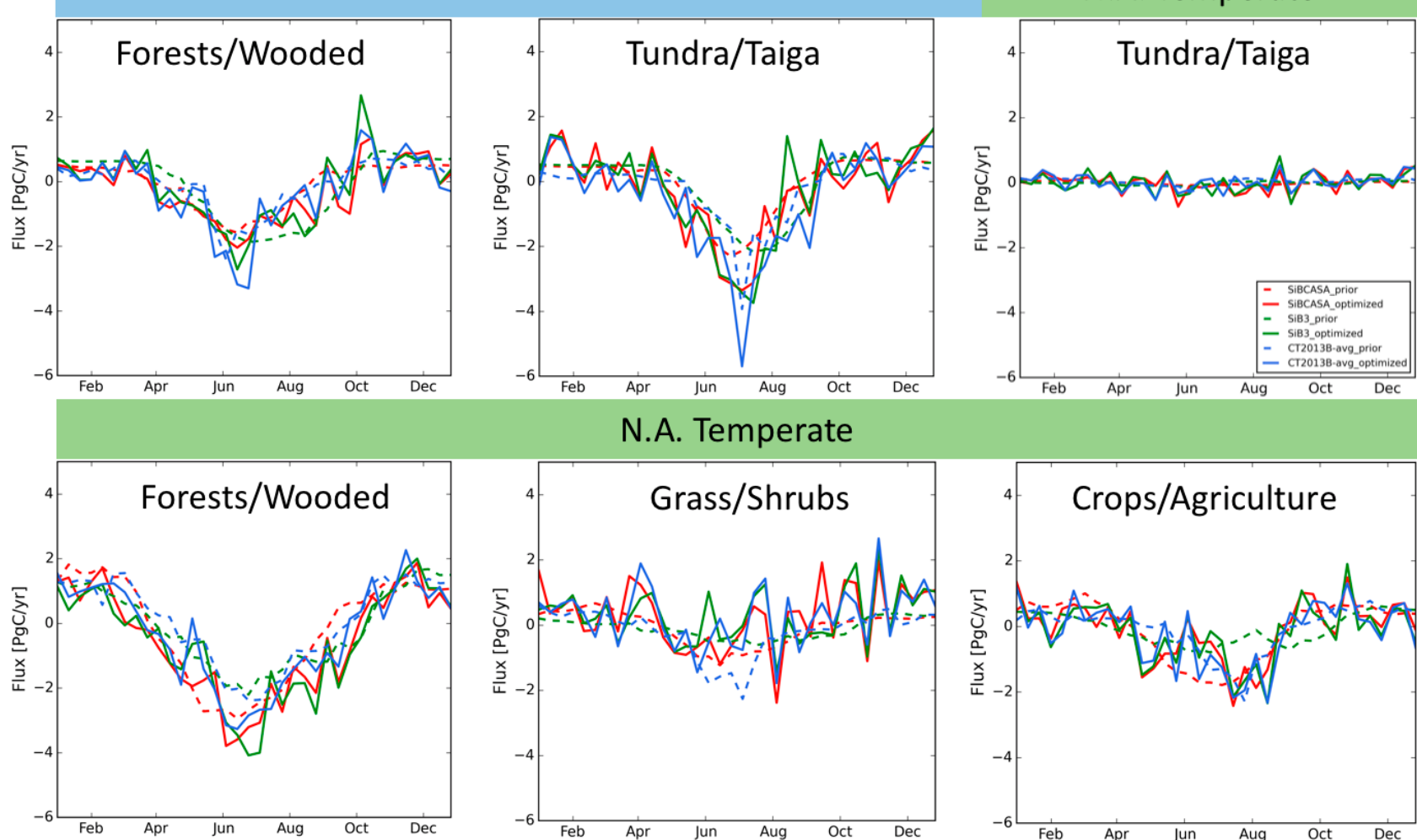

\section{N.A. Temperate}
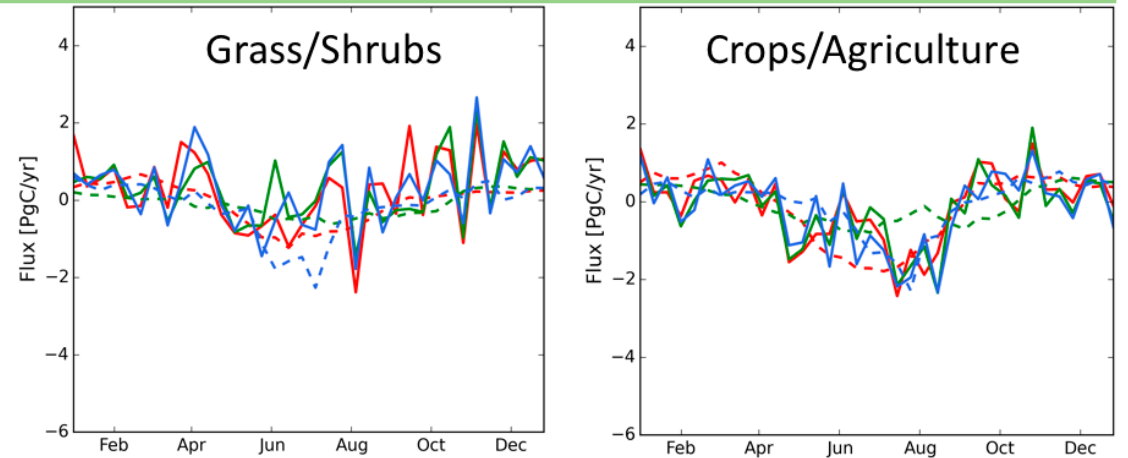

Figure S3. The time series of prior and optimized fluxes of major aggregated ecoregions for the runs with three different prior biosphere fluxes (SiBCASA, SiB3 and CT2013B-avg). The optimized fluxes at most ecoregions are closer to each other than the prior fluxes, except for the Boreal Tundra/Taiga during July-October, which could be due to sparse observations in that region. 


\section{Tests with modified SiBCASA prior fluxes}
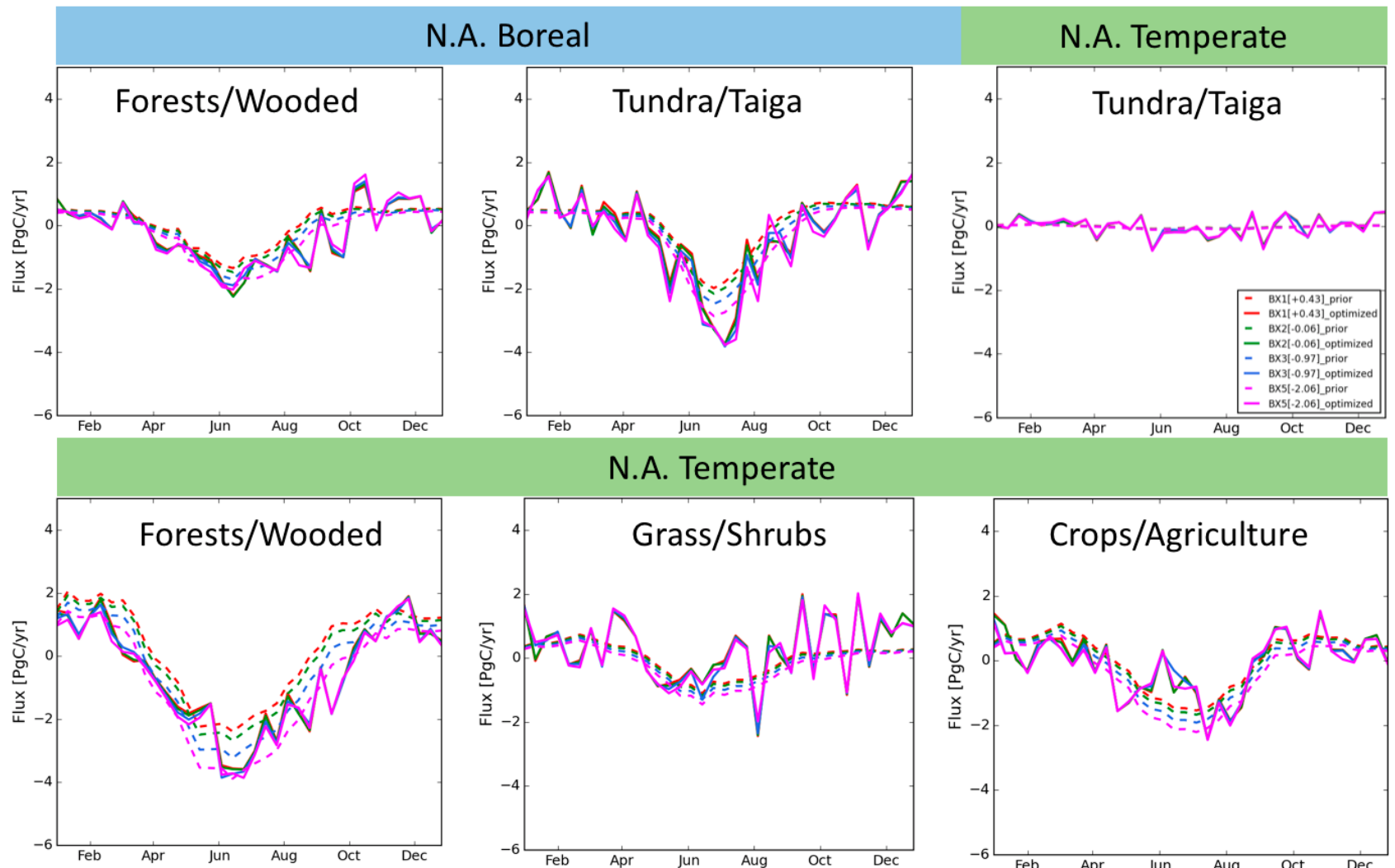

\section{N.A. Temperate}
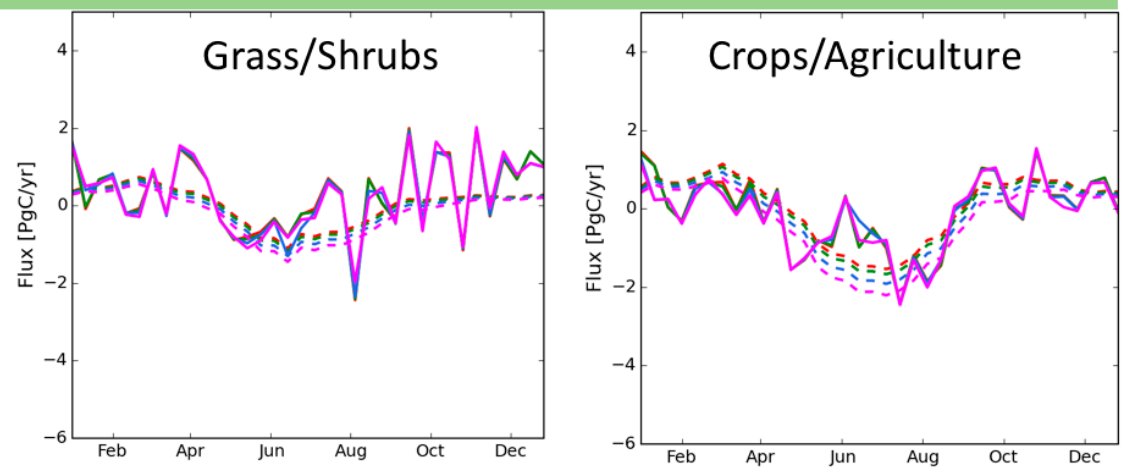

Figure S4. The time series of prior and optimized fluxes of major aggregated ecoregions for the runs with three different modified SiBCASA prior fluxes. The optimized fluxes largely agree with each other for all ecoregions despite the large differences in prior fluxes. A better agreement in the optimized fluxes is seen than from different biosphere models shown in Figure S3. This comparison indicates the spatial distribution of prior fluxes has a significant impact on the optimized fluxes. 